This is the peer reviewed version of the following article: "Wang, L., Cui, L., Sánchez-Soto, M., Shou, W., Xia, Z., and Liu, y. (2018) Highly flame retardant melamine-formaldehyde cross-linked cellulose nanofibrils/sodium montmorillonite aerogels with improved mechanical properties, vol. 303, núm. 10: 1800379-1-9." which has been published in final form at [doi: 10.1002/mame.201800379]. This article may be used for non-commercial purposes in accordance with Wiley Terms and Conditions for Self-Archiving." 


\title{
Highly Flame Retardant Melamine-formaldehyde Cross-linked Cellulose Nanofibrils/Sodium Montmorillonite Aerogels with Improved Mechanical Properties
}

\author{
Liang Wang ${ }^{1 *}$, Lingyan Cui ${ }^{1}$, Miguel Sánchez-Soto ${ }^{2}$, Wan Shou ${ }^{3}$, Zhaopeng Xia ${ }^{1}$ \\ and Yong Liu ${ }^{1 *}$
}

1. School of Textiles, Tianjin Polytechnic University, Binshui West Road 399, Xiqing Distinct, 300387, Tianjin, China.

2. Centre Catalá del Plástic, Universitat Politécnica de Catalunya, Barcelona Tech., Colom 114, 08222 Terrassa, Spain.

3. Department of Mechanical \& Aerospace Engineering, Missouri University of Science and Technology, Rolla, MO. 65409, USA.

*Corresponding authors:

Dr. Liang Wang, liangwang@tjpu.edu.cn; Prof. Yong Liu, liuyong@tjpu.edu.cn. Tel: $+86-22-83955298$.

Address: School of Textiles, Tianjin Polytechnic University, No.399 Binshui West Road, Xiqing District, Tianjin 300387, China.

\begin{abstract}
A facile cross-linking strategy to construct flame retardant cellulose nanofibril (CNF)/sodium montmorillonite (MMT) aerogels with improved mechanical properties by incorporating melamine-formaldehyde (MF) resins into precursor suspensions followed by a freeze-drying process was reported in this work. Scanning electron microscopy images indicated that MF cross-linking did not significantly change the microstructures of $\mathrm{CNF}$ and CNF/MMT aerogels. However, the
\end{abstract}


crosslinking improved the materials' mechanical and flame properties. By incorporating $50 \mathrm{wt} \%$ of $\mathrm{MF}$, the compression moduli and compressive stress of $\mathrm{CNF}$ aerogels increased by $316 \%$ and $114 \%$, respectively. The limiting oxygen index (LOI) value of CNF aerogels also increased from $17.1 \%$ to $23.4 \%$. Further addition of MMT increased the CNF aerogels' LOI value to $57 \%$ and increased the maximum decomposition temperature by nearly $20{ }^{\circ} \mathrm{C}$. This occurred because MMT and MF induced a synergistic effect which improved the flame retardant properties of the CNFs aerogels. In CNF/MMT composite aerogels, the introduction of $34 \mathrm{wt} \%$ of MF led to a $54.6 \%$ reduction of the peak of heat release rate and a $53.2 \%$ decrease in total heat release. CNF aerogels made from sustainable feedstocks with excellent mechanical properties and high flame retardancy, like those discussed in this paper, show promise as fire resistant biofoams.

\section{Introduction}

Aerogels were pioneered by Kistler in the early 1930s. ${ }^{[1]}$ These materials are characterized by their ultralow density and high porosity. ${ }^{[2]}$ Since Kistler's discovery, aerogels have been developed using various matrices including silica, ${ }^{[3]}$ carbon, ${ }^{[4-6]}$ metals, ${ }^{[7]}$ ceramics, ${ }^{[8]}$ and polymers. ${ }^{[9]}$ Recent research has studied the use of natural cellulose as a matrix because cellulose is sustainable and biodegradable. In particular, cellulose nanofibrils (CNFs) have been a focus of the past decade of research. ${ }^{[10-13]}$ CNF aerogels combine high specific surface area and porosity with excellent biocompatibility and biodegradability, which makes these materials good candidates 
for use in applications like wastewater treatment, ${ }^{[14]}$ drug sustained release, ${ }^{[15]}$ catalyst carrier, ${ }^{[16]}$ and flexible devices, ${ }^{[17]}$ etc.

CNF aerogels are excellent thermal insulators due to their high porosity. ${ }^{[18]}$ Nevertheless, cellulose is a highly flammable material. This flammability severely limits the usage of CNF aerogels in many important high-temperature applications. To overcome this drawback, various methods have been used to improve the flame retardant properties of CNF aerogels. Yuan et al ${ }^{[19]}$ prepared silica-CNF hybrid aerogels that exhibited excellent flame retardant properties and displayed selfextinguishment after ignition when silica content was higher than 33.6\%. Javadi and coworkers ${ }^{[20]}$ enhanced the thermal stability and flame retardancy of CNFs aerogels by adding graphene oxide. Yang et al constructed fire resistant CNF aerogel by encapsulating two-dimensional molybdenum disulfide $\left(\mathrm{MoS}_{2}\right)$ nanosheets. The $\mathrm{CNF} /$ $\mathrm{MoS}_{2}$ aerogels had a limiting oxygen index of $34.7 \%$ and released super low amount of combustive heat $\left(0.4 \mathrm{MJ} / \mathrm{m}^{2}\right){ }^{[21]}$ Magnesium hydroxide $(\mathrm{MH})$ and aluminum hydroxide $(\mathrm{AH})$ nanoparticles have also been synthetized in-situ within CNF aerogels to improve the material's flame resistance, respectively. ${ }^{[22,23]}$ Generally, these modification processes are complicated, harmful to the environment, or expensive. However, the addition of flame retardant agents generally does not increase the mechanical properties of the aerogels. This is because the primary intermolecular force at work in these materials is the Van de Walls force. Based on these conditions and limitations, it is reasonable to expect that future research will focus on the development of low-cost processes that improve both the mechanical and fire 
properties of CNF aerogels.

Melamine-formaldehyde (MF) resin has been widely used as a gas-phase flame retardant additive in polymers. ${ }^{[24-26]}$ In acidic media and at specific temperatures, MF resins can interact with the hydroxyl groups on the surface of the cellulose. ${ }^{[27]}$ However, MF cross-linked CNF aerogels have not been widely studied. This study was designed to simultaneously improve the mechanical properties and flame retardancy of CNF aerogels via cross-linking. In this process, CNFs with abundant surface hydroxyl groups reacted with MF resins. CNF and CNF/MMT aerogels were prepared via a freeze-drying process as described previously. ${ }^{[28]} \mathrm{MF}$ resins were added to the precursor suspensions and then freeze-dried. The resulting CNF based aerogels were then heated to yield MF cross-linked CNF aerogels. The morphologies, mechanical properties, thermal stabilities, and burning behaviors of these materials were investigated.

\section{Experimental Section}

\subsection{Materials}

Cellulose nanofibrils (CNFs) with a diameter of 4-10 nm and a length of 1-3 $\mu \mathrm{m}$ were supplied by Guilin Qihong technology Co., Ltd (Guilin, China). Sodium montmorillonite $\left(\mathrm{Na}^{+}-\mathrm{MMT}\right.$, Nanomer PGW grade) was bought from Nanocor (USA). Melamine-formaldehyde resin (MF, Permutex XR-9181) was purchased from Starr Paint Co., Ltd (Suzhou, China) as a cross-linking agent. Phosphoric acid of purity not less than $85 \%$ was supplied by Tianjin Sailboat Chemical Reagent Technology Co., 
Ltd (Tianjin, China). All chemicals were used without further purification.

\subsection{Preparation of Pure CNF Aerogels}

CNF suspensions (1 wt $\%$ ) were prepared by dispersing $1 \mathrm{~g}$ dry CNF in $100 \mathrm{~mL}$ of deionized (DI) water via mechanically stirring at $1200 \mathrm{rpm}$ for 5 hours. The transparent precursors were then transferred into polypropylene molds followed by freezing in an ethanol/liquid nitrogen bath at $-116{ }^{\circ} \mathrm{C}$ for 30 minutes. The freezedrying procedure was conducted in a lyophilizer (Scientz-10N, China) for 72 hours under vacuum of $1 \mathrm{~Pa}$ and a condenser temperature of $-55^{\circ} \mathrm{C}$.

\subsection{Preparation of CNF/MMT Composite Aerogels and Cross-linked Aerogels}

The CNF composite aerogels were prepared following the scheme shown in Figure 1. MMT (1g) was dispersed in $50 \mathrm{~mL}$ of DI water using homomixer (ART-D9, Germany) at $20000 \mathrm{rpm}$ for 10 minutes to obtain a $2 \mathrm{wt} \%$ clay suspension. Then, this MMT suspension was combined with $100 \mathrm{~mL}$ of the $1 \%$ CNF suspension described in section 2.2. The mixture was mechanically stirred for 1 hour until homogenous. The $\mathrm{pH}$ of the homogenous suspension mixture was adjusted to 4 via dropwise addition of phosphoric acid.

Variable amounts of MF were added to the homogenous suspension to crosslink the CNF. The compositions of the samples that were studied in this test are detailed in Table 1. After the MF was added, the suspensions were mixed until homogenous. The resultant homogeneous mixtures were frozen and lyophilized. Finally, the aerogels 
containing MF were placed in an oven at $120^{\circ} \mathrm{C}$ for 7 hours to achieve cross-linking. The obtained samples were identified as CNFxMMTyMFz, where $x, y$ and $z$ represents corresponding solid mass percentage of each component in the composite aerogel, respectively.

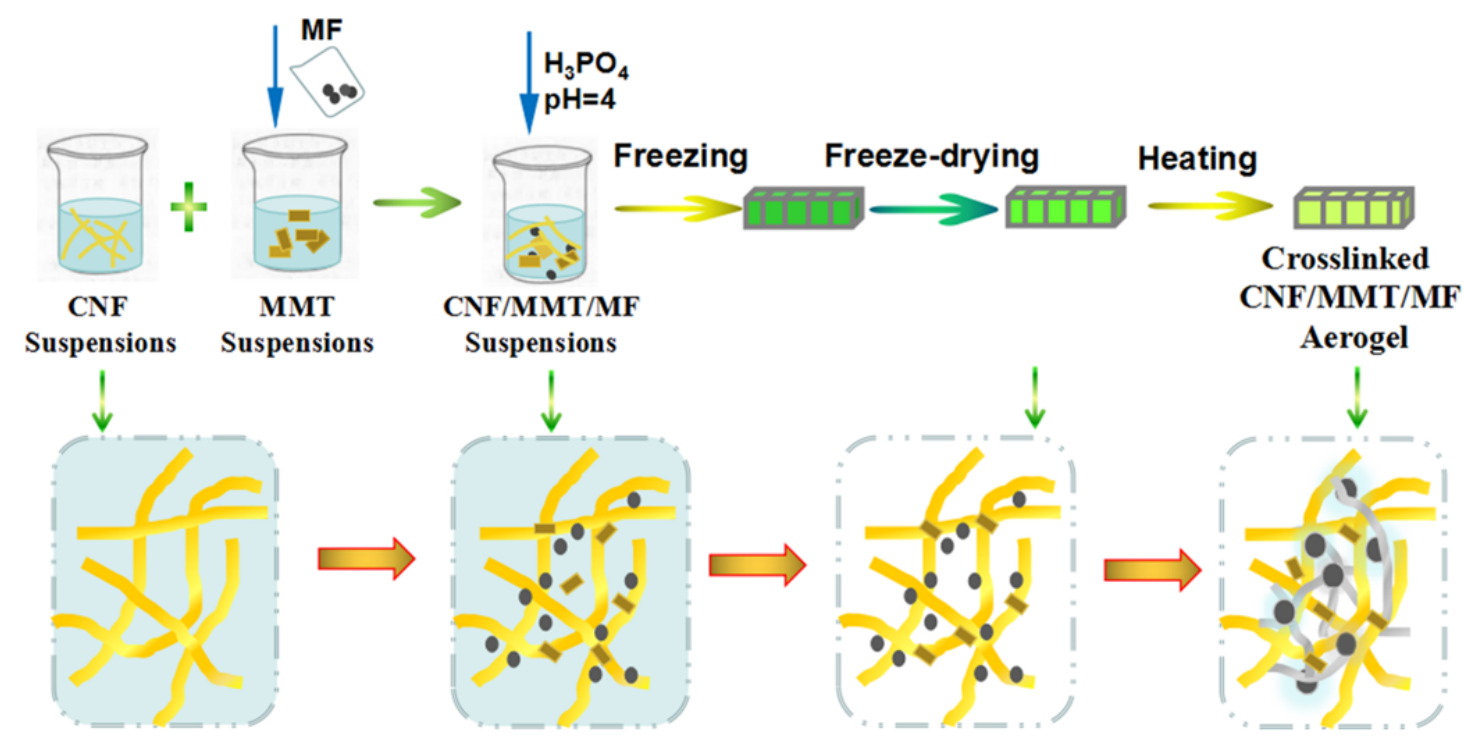

Figure 1.

\subsection{Characterization}

The chemical structures of aerogels were studied using a Nicolet iS50 Fourier infrared spectrophotometer in the attenuated total reflectance mode (FTIR-ATR, Thermo Fisher, USA). Each sample had a spectral result based on 30 scans with a resolution higher than $0.09 \mathrm{~cm}^{-1}$ across a wavenumber interval between 4000 and 400 $\mathrm{cm}^{-1}$

The microstructures of aerogels were characterized using a scanning electron microscope (SEM, Hitachi-TM3030, Japan) at an acceleration voltage of $15 \mathrm{kV}$. Before observing, samples were cyro-fractured and sputter coated with a gold layer.

The apparent densities of aerogels were calculated by the division of mass to 
volume of cylindrical samples. Five samples were used to evaluate each composition. Compression tests were performed using a Hongda (China) universal testing machine using a load cell of $5 \mathrm{kN}$ following the ISO 604 standard. The crosshead rate and maximum strain were set to $5 \mathrm{~mm} / \mathrm{min}$ and $70 \%$, respectively. Five samples were used to evaluate each composition.

Thermogravimetric analysis (TGA) was carried out to evaluate the thermal stability of aerogels using a STA449F3 thermogravimetric analyzer (Netzsch, Germany). The experiments were conducted under nitrogen atmosphere with a heating rate of $10{ }^{\circ} \mathrm{C} / \mathrm{min}$ from $30{ }^{\circ} \mathrm{C}$ to $800{ }^{\circ} \mathrm{C}$.

The limiting oxygen index (LOI) was measured using an oxygen index meter (ATSFAAR 20008600, Italy) according to ASTM D2863-2009. Each specimen was cut into a $120 \times 10 \times 10 \mathrm{~mm}$ section and placed in a combustor where the oxygen content of the atmosphere was adjusted to find the lowest oxygen concentration that could support sustained burning. Limiting oxygen index was expressed as a volume percentage.

The vertical burning test (UL-94) was performed on a HVUL2 instrument (Atalas, USA) according to GT/T 8333-2008. The size of all samples used for testing was $125 \times 10 \times 10 \mathrm{~mm}$.

The combustion behaviors of the $\mathrm{CNF} / \mathrm{MMT} / \mathrm{MF}$ aerogels were analyzed using a cone calorimeter (FIT9116061, England) following the ISO 5660 procedure. Square samples $(100 \times 100 \mathrm{~mm})$ with an average thickness of $10 \mathrm{~mm}$ were mounted in a steel support and exposed to an external heat flux of $50 \mathrm{~kW} / \mathrm{m}^{2}$. 


\section{Results and Discussion}

\subsection{FTIR Analysis}
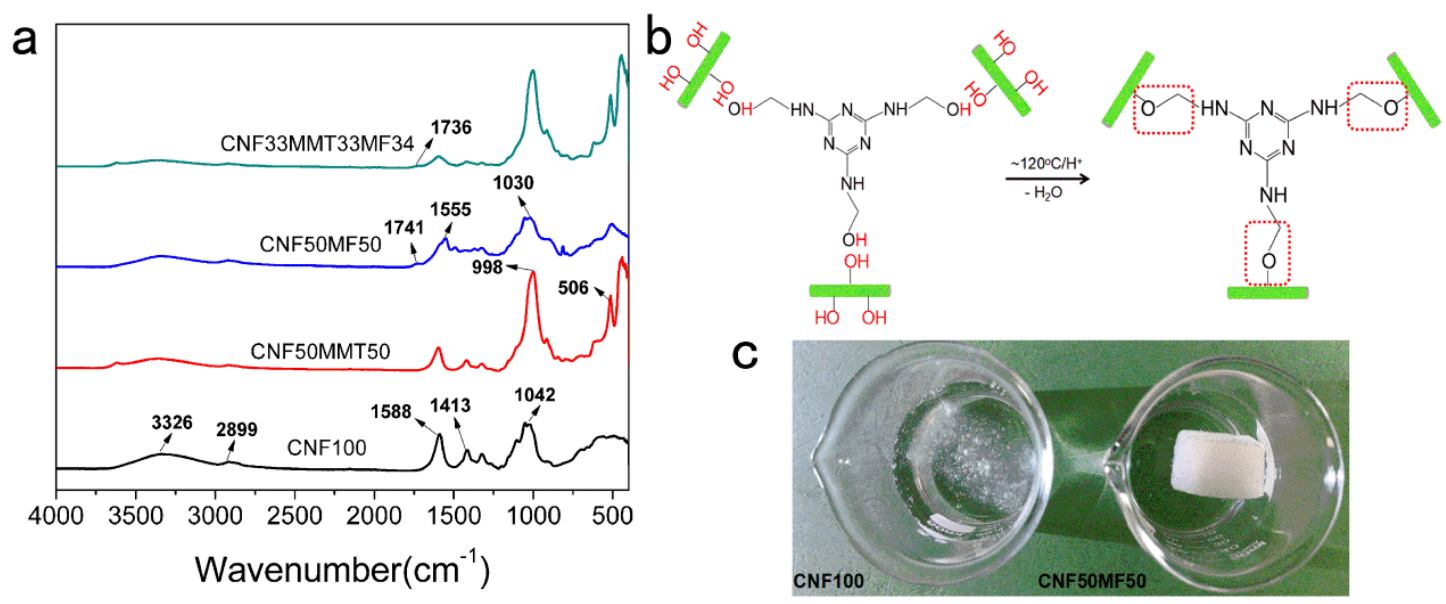

Figure 2.

FTIR analysis was conducted to confirm the cross-linking reaction of CNFs and MF.

The spectra obtained from the aerogels are shown in Figure 2a. The characteristic peaks of pure CNF aerogel are located at $3326 \mathrm{~cm}^{-1}, 1588 \mathrm{~cm}^{-1}$ and $1042 \mathrm{~cm}^{-1}$, which correspond to bonded $\mathrm{OH}$ stretching, $\mathrm{OH}$ bending and the stretching vibration of $\mathrm{COC}$ bonds that bridge the glucose units, respectively. The other two bands at $2899 \mathrm{~cm}^{-1}$ and $1413 \mathrm{~cm}^{-1}$ are associated with asymmetrical $\mathrm{CH}_{2}$ stretching and scissoring, respectively. ${ }^{[29,30]}$ With the addition of the MMT, new peaks appear at $506 \mathrm{~cm}^{-1}$ and $998 \mathrm{~cm}^{-1}$, which are associated with the clay's AlO and SiOSi groups respectively. ${ }^{[31]}$ MF modified CNF aerogels displayed a new band at $1741 \mathrm{~cm}^{-1}$ due to the $\mathrm{C}=\mathrm{O}$ groups of free-floating formaldehyde molecules. ${ }^{[32]}$ The band at $1555 \mathrm{~cm}^{-1}$ was assigned to the $1,3,5$-s-triazing ring mode of melamine. ${ }^{[33]}$ The peak of COC originally located at $1042 \mathrm{~cm}^{-1}$ shifted to $1030 \mathrm{~cm}^{-1}$ and the reflection peak of $\mathrm{OH}\left(3326 \mathrm{~cm}^{-1}\right)$ became 
lower on intensity, suggesting that hydroxyl groups of CNFs reacted with MF as depicted in Figure 2b. To further prove this cross-linking reaction, the swelling behavior of CNF100 and CNF50MF50 in DI water were observed. The skeleton of CNF100 collapsed because the hydrogen bonding between CNFs was broken in water. MF cross-linked sample (CNF50MF50) absorbed water and turned into a stable hydrogel, as seen in Figure 2c. The networks formed between the CNFs created blocked skeletons of aerogels. Increasing the water temperature to $100{ }^{\circ} \mathrm{C}$, no significant change occurred on the appearance of obtained hydrogel. This phenomenon indicated that MF effectively cross-linked the CNFs.

\subsection{Morphologies of Aerogels}

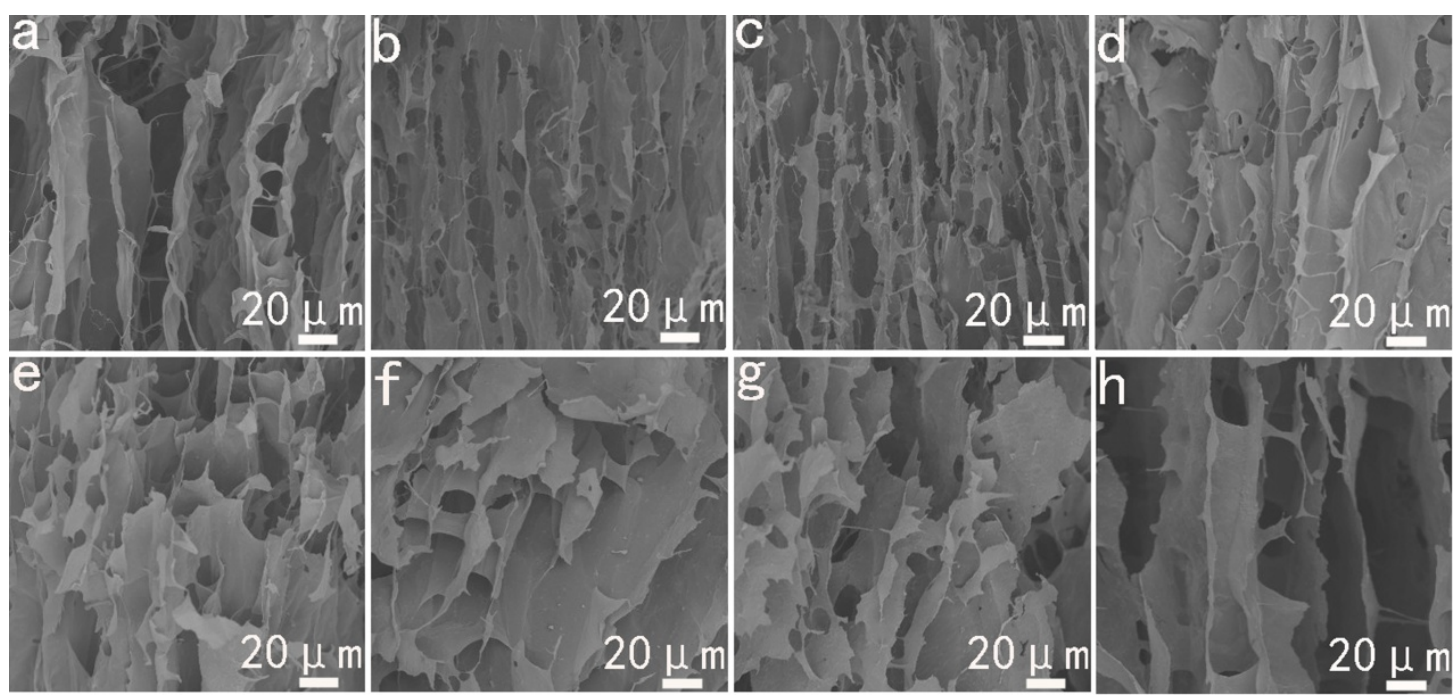

Figure 3.

Neat CNF aerogels exhibited a typical lamellar structure. As seen in Figure 3a, pure

CNF aerogels have oriented channels that were created by the ice crystals' growth 
path during freezing. When MF was added to crosslink the CNFs, the increased viscosity of the suspension led to a reduced rate of ice growth. Because of this, interlayer spacing became narrower and the layers became thicker. This is shown in figures $3 \mathrm{~b}$ and $3 \mathrm{c}$. Increasing MF amount in CNF aerogel (CNF40MF60), the quantity of polymeric struts connecting the layers decreased as seen in figure $3 \mathrm{~d}$. This is possibly induced by the self-polymerization of excessive MF, which was widely reported in works about MF coating at a similar condition. ${ }^{[34-36]}$

MMT introduction led to the formation of the three dimensional network structure shown in figure $3 \mathrm{e}$. The CNFs formed hydrogen bonds with the clay platelets and these bonds formed the cell walls of the aerogels. ${ }^{[37]}$ The addition of MMT increased the viscosity of precursor solutions in the same way that the addition of MF did. This increased viscosity hindered the growth of ice crystals. The mobility of CNFs was limited due to this high viscosity and secondary crystallization occurred. $^{[38]}$ Low levels of MF did not significantly affect the structure of $\mathrm{CNFs} / \mathrm{MMT}$ aerogels as shown in figures $3 \mathrm{f}$ and $3 \mathrm{~g}$. However, higher levels of MF (CNF28MMT28MF44) led to a structure with larger pores and lower number of connecting struts as shown in figure $3 \mathrm{~h}$. This could have occurred due to selfpolymerization of MF which lead to a decrease of the viscosity of the precursor suspension. 


\subsection{Compressive Properties}
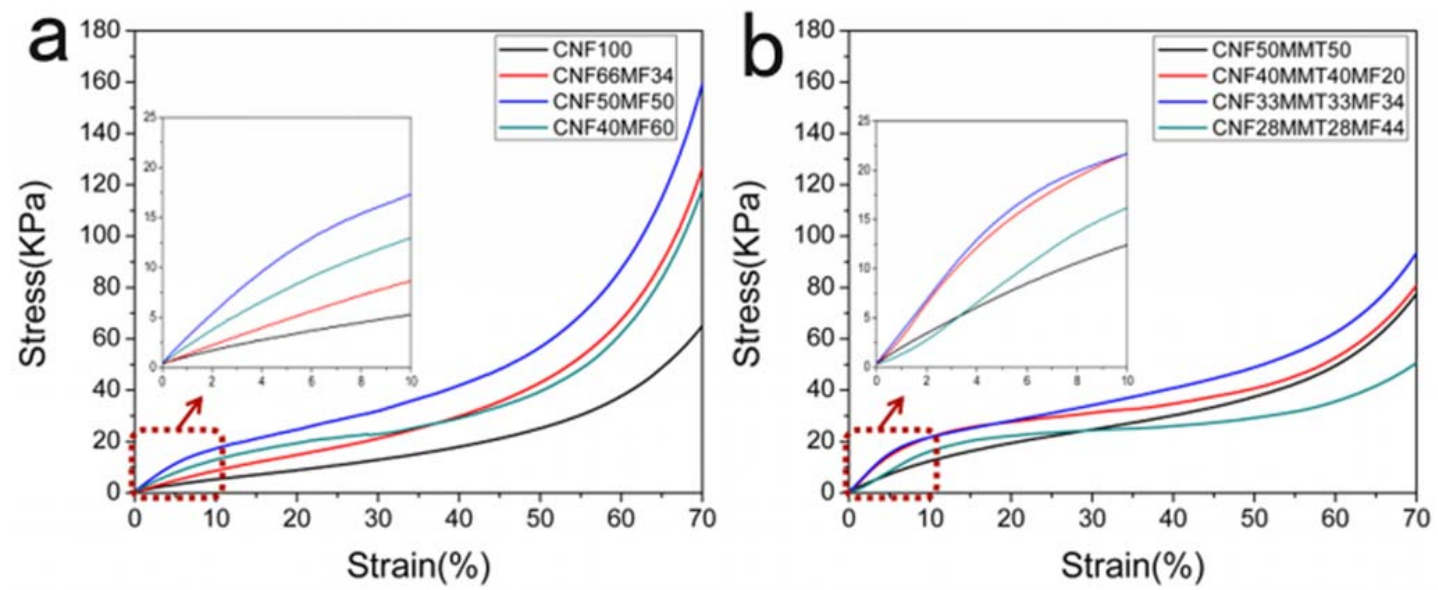

Figure 4.

Compressive stress-strain curves of aerogels are shown in figures $4 \mathrm{a}$ and $4 \mathrm{~b}$. All of the samples displayed typical elastic-plastic foam behavior. The parameters defining the compressive mechanical behavior, compressive moduli $(\mathrm{E})$, stress at $10 \%$ of strain $\left(\sigma_{10 \%}\right)$ and stress at $70 \%$ of strain $\left(\sigma_{70 \%}\right)$ are summarized in Table 2 . Stress at $10 \%$ of strain $\left(\sigma_{10 \%}\right)$ was thought to be the yield stress $\left(\sigma_{\mathrm{y}}\right)$ of aerogels according to the compressive curves. The specific moduli $\left(\mathrm{E}_{\mathrm{s}}\right)$ and specific yield stress $\left(\sigma_{\mathrm{sy}}\right)$ were also calculated and are included in Table 2.

The samples of CNF100 were easily compressed with a modulus of $65.59 \mathrm{kPa}$ and yield strength of $65.15 \mathrm{kPa}$. By incorporating $50 \%$ of $\mathrm{MF}$, the compression moduli and compressive stress $\left(\sigma_{70 \%}\right)$ of CNF50MF50 were increased 3.16 and 1.44 times, respectively. Additionally, both the $\mathrm{E}_{\mathrm{s}}$ and $\sigma_{\mathrm{sy}}$ were increased with the addition of MF. This is because cross-linking between CNFs and MF enhanced the structural integrity of aerogels, making the materials more resistant to deformation under applied stress. ${ }^{[39]}$ However, higher levels of MF reduced the mechanical properties of 
CNF-based aerogels (sample CNF40MF60). This may have been caused by the selfpolymerization of MF. Hydrophobic MF polymer could decrease cross-link quantity and break the interfacial bonding between CNFs. This effect is shown in Figure 3d.

A similar phenomenon was observed in CNF/MMT composite aerogels when MF was added. CNF33MMT33MF34 had a specific modulus of $18.2 \mathrm{MPa}$ and a specific yield stress of 5.5 MPa, which were higher than the corresponding values of CNF50MMT50. These increases indicate that the cross-linking effect of MF also works in $\mathrm{CNF} / \mathrm{MMT}$ composite aerogels. The mechanical properties of foam-like aeogels also depend on their apparent density. Greatest compressive stress $\left(\sigma_{70 \%}\right)$ was observed on CNF50/MF50 that has highest apparent density. First, CNF concentration in corresponding suspensions of $\mathrm{CNF} / \mathrm{MF}$ aerogels is higher than the one for preparing CNF/MMT/MF composite aerogels, as seen in Table 1. Second, Clay existence in aerogels decreases the volume shrinkage of composite aerogels.

\subsection{Thermal Stability}
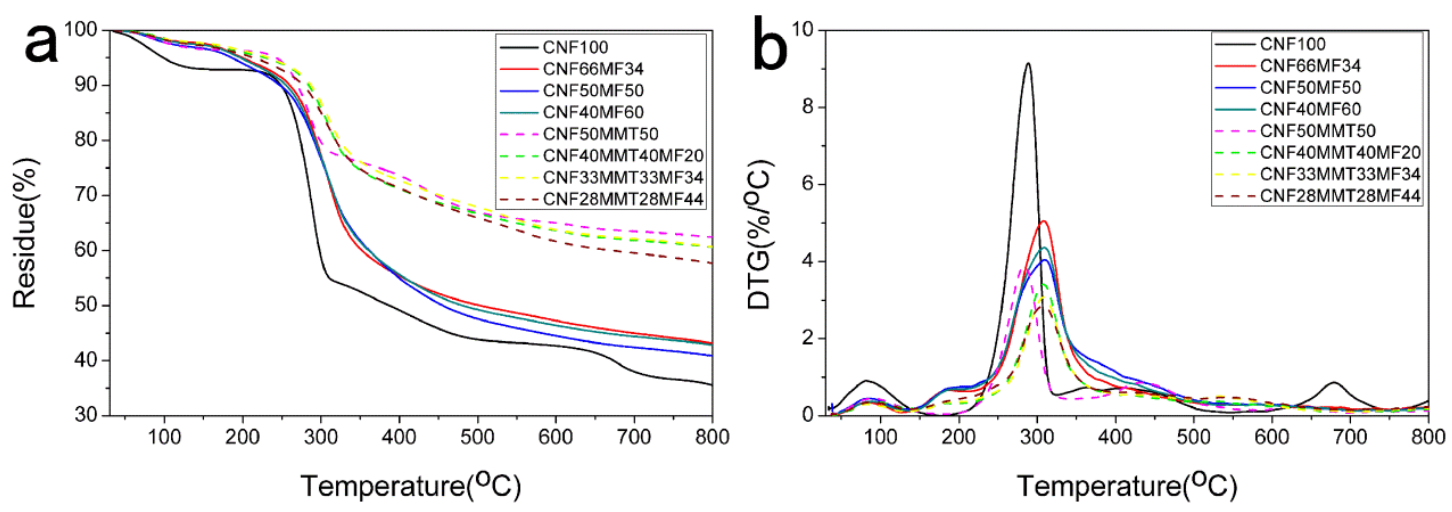

Figure 5.

TGA weight loss data and corresponding DTG curves of aerogels are shown in Figure 
5. The relevant parameters, including the onset decomposition temperature $\left(\mathrm{T}_{\mathrm{d} 5 \%}\right)$, maximum decomposition temperature $\left(\mathrm{T}_{\mathrm{dmax}}\right)$, maximum mass decomposition rate $\left(\mathrm{dW} / \mathrm{dT}_{\max }\right)$ and residue amount $\left(\mathrm{W}_{\mathrm{R}}\right)$ are summarized in Table $3 . \mathrm{T}_{\mathrm{d} 5 \%}$ was given as the temperature at which $5 \%$ weight beyond total loss of water occurred after $120{ }^{\circ} \mathrm{C}$. As seen in Figure 4a, all the samples displayed similar decomposition patterns during two main steps of weight loss. First, a slight weight loss occurred before $200{ }^{\circ} \mathrm{C}$ due to moisture removal in CNFs and small molecule volatilization in MF resins. Second, a sharp mass loss occurred when the temperature surpassed $250{ }^{\circ} \mathrm{C}$. It was attributed to the decomposition of molecular chains of CNFs or MF.

The addition of MF resulted in slightly lower $\mathrm{T}_{\mathrm{d} 5 \%}$ values for $\mathrm{MF}$ cross-linked CNF aerogels compared to pure CNF aerogels. This is because small molecules from MF resins have lower decomposition temperatures than CNFs. Despite lower $\mathrm{T}_{\mathrm{d} 5 \%}$ values, $\mathrm{CNF} / \mathrm{MF}$ composites exhibit higher maximum decomposition temperatures, lower maximum weight loss rates and larger residue amounts than pure CNF aerogels. By incorporating $50 \% \mathrm{MF}, \mathrm{T}_{\mathrm{dmax}}$ increased by $\sim 20{ }^{\circ} \mathrm{C}$ and maximum weight loss rate decreased from 9.2 to 4.0. These changes are attributed to the formation of cross-links between CNFs and MF. ${ }^{[39]}$

After adding $50 \mathrm{wt} \%$ of MMT to CNF aerogels, $\mathrm{T}_{\mathrm{d} 5 \%}$ increased by nearly $10{ }^{\circ} \mathrm{C}$ and maximum weight loss rate dropped by $57.6 \%$ compared to pure CNF aerogels. This is because the added MMT thermally insulated the CNFs and protected them from rapid thermal decomposition. ${ }^{[40]}$ However, MMT addition caused a mild decrease in $T_{d m a x}$, possibly related to the higher thermal conductivity resulting from 
denser and more compact structure of the aerogels ${ }^{[28]}$. When MF was added into CNF/MMT composite aerogels, $\mathrm{T}_{\mathrm{dmax}}$ increased by over $20{ }^{\circ} \mathrm{C}$ and $\mathrm{dW} / \mathrm{dT}_{\max }$ monotonically decreased with the MF content in aerogels. These improvements were attributed to the cross-linked structure of the aerogel as well as the positive effect of MMT on char formation.

\subsection{Combustion Behavior}

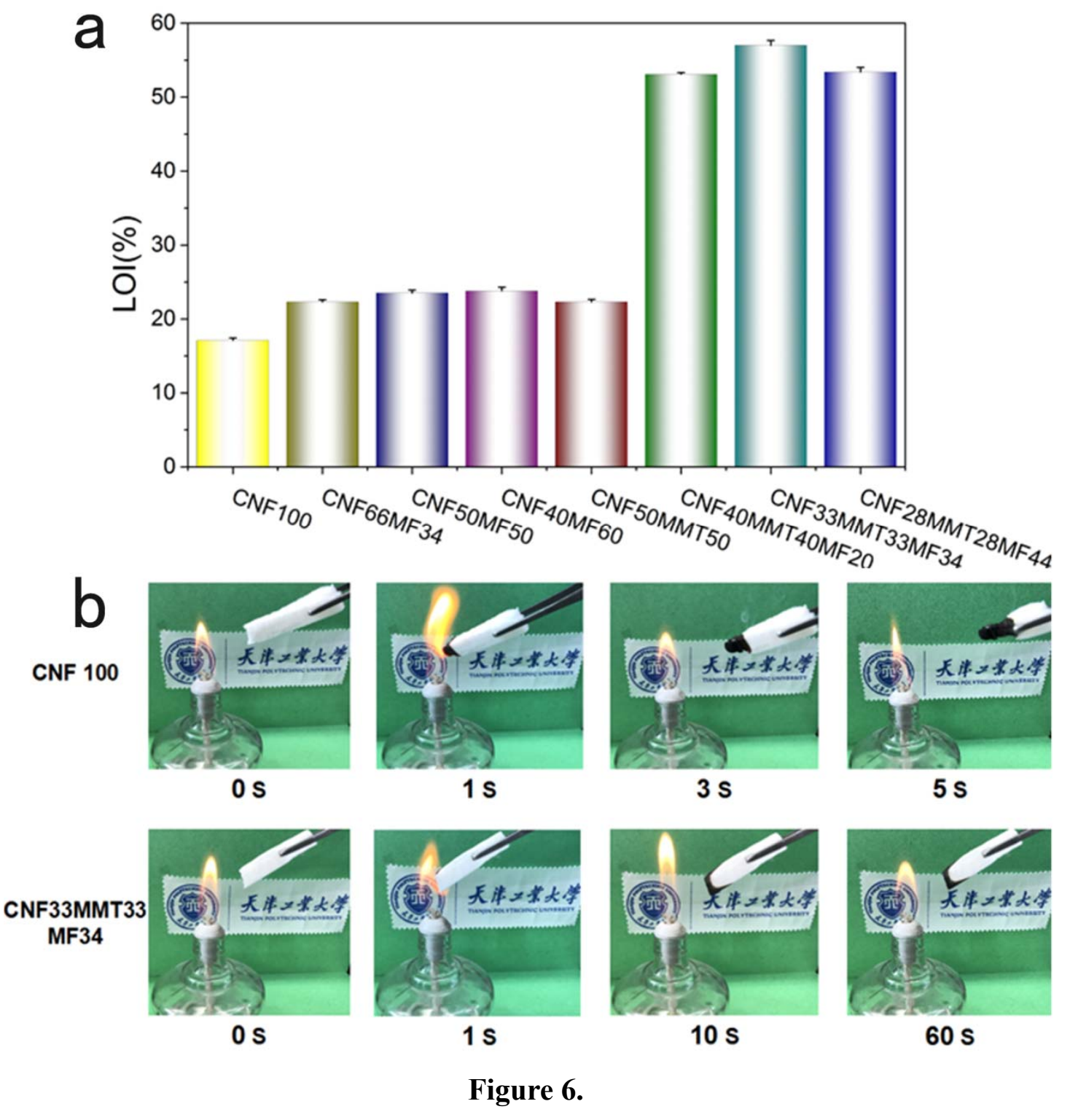


Limiting oxygen index (LOI) tests and UL-94 tests were conducted to compare the flammability of the pure CNF aerogels and CNF composite aerogels. The corresponding results are shown in Figure 6a and Table 4, respectively. Neat CNF aerogels were the most flammable with a low LOI value of $17.1 \%$ and with no UL-94 rating. The addition of both MMT and MF caused minor increase of LOI of CNFbased aerogels. Meanwhile, these samples did not show UL-94 rating. MMT is wellknown for its contribution to charring. However, the protective barrier formed by MMT in the fire region only slows the flame spread. MMT does not significantly reduce the fire load, ignitability, or flammability of the material. ${ }^{[41]}$ Consequently, the sample of CNF50MMT50 also showed a relatively low LOI value of $22.3 \%$. LOI values increased by MF cross-linking primarily because MF combustion produces gasses which have a diluting effect. ${ }^{[39]}$ Low contribution of MF to char formation resulted in a slight increase of 5.2-6.7\% in the LOI value of MF-modified aerogels.

When MF and MMT were simultaneously used to modify CNFs based aerogels, the corresponding composite aerogels reached $\mathrm{V}-0$ rating with much more pronounced improvement in LOI values. When each component accounted $1 / 3$ of weight of aerogel (CNF33MMT33MF34), the LOI value of aerogels increased to $57 \%$. It was also observed that the aerogels showed a self-extinguishing property and merely shrank a little after $60 \mathrm{~s}$ of ignition by an alcohol burner, as shown in Figure 6b. These suggest a synergistic effect between MMT and MF's impacts on the flame retardant properties of CNF aerogels. However, when the MF content was further increased to CNF28MMT28MF44 a slight decrease in LOI values was observed. This 
phenomenon could be due to self-polymerization of MF which reduced the amount of free melamine molecules that can decompose at lower temperatures.
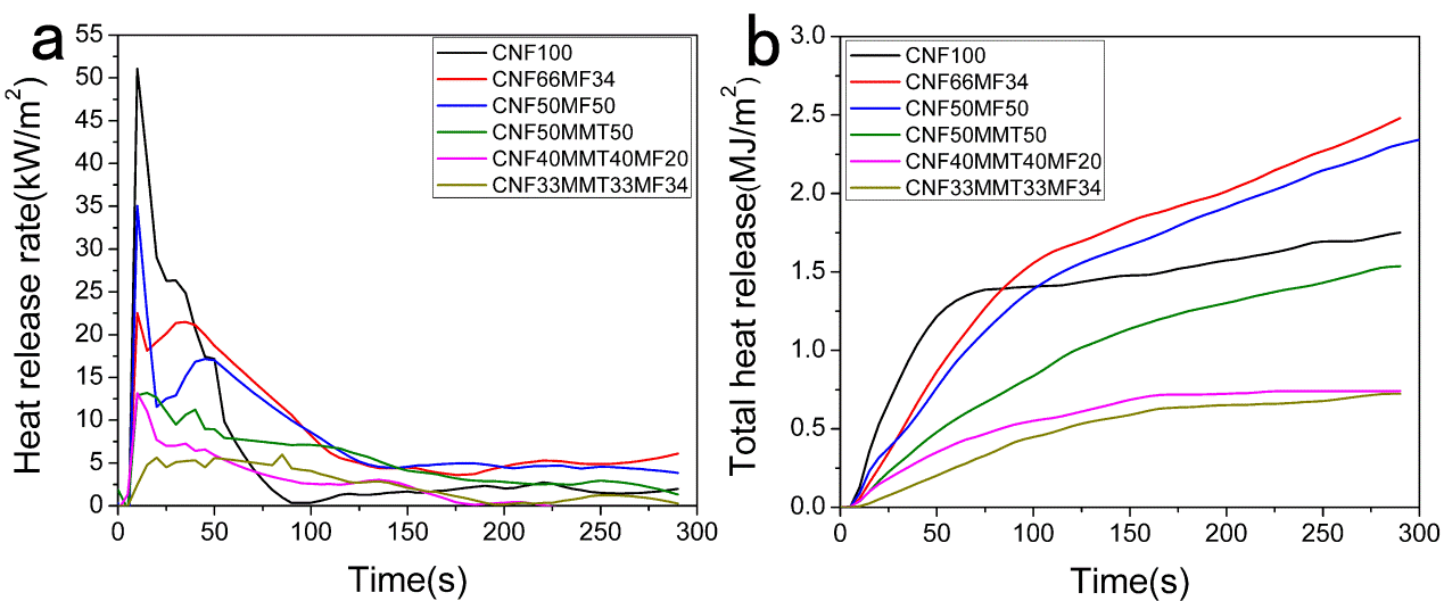

Figure 7.

The combustion of these aerogels was also investigated via cone calorimetry tests which mimic the combustion behavior of a material in a real fire scenario. The heat release rate and total heat release of the samples are exhibited in Figures $7 \mathrm{a}$ and $7 \mathrm{~b}$, respectively. Corresponding quantitative information including peak of heat release rate (PHRR), time to PHRR (TTPHRR), total heat release (THR), fire growth rate (FGR) and residue amount $\left(\mathrm{W}_{\mathrm{R}}\right)$ are listed in Table 5 .

Neat CNFs aerogels displayed a typical combustion behavior which has been described in previous works. ${ }^{[42]}$ Its HRR pattern exhibited a sharp peak after burning for $10 \mathrm{~s}$ with a PHRR value of $51.05 \mathrm{~kW} / \mathrm{m}^{2}$. This behavior indicates that pure CNF aerogels burn quickly and release large amounts of heat in a short time. After crosslinking with $34 \mathrm{wt} \%$ of MF, PHRR and FGR decreased by $28.54 \mathrm{~kW} / \mathrm{m}^{2}$ and 2.86 $\mathrm{W} / \mathrm{s}$, respectively. This decline was due to the decomposition of MF which releases 
$\mathrm{NH}_{3}$. The $\mathrm{NH}_{3}$ dilutes the fuel gas and hinders the spread of flame. MF cross-linked aerogels exhibit higher THRs than the pure ones. This may be due to the burning of MF which also contributes to heat release. Increased amounts of MF did not improve the flame retardant properties of the aerogels significantly due to the decrease of free melamine molecules as discussed previously.

The addition of MMT reduced the PHRR, THR and FGR of cellulose aerogels, which was expected based on prior research. ${ }^{[43]}$ This is attributed to the clay enrichment of the sample surface during heating, ${ }^{[4]}$ protecting the substrate material. LOI tests showed that MMT and MF had a synergistic flame retardant effect on CNFs aerogels. This phenomenon is also observed in cone calorimetry tests. Compared to sample CNF50MMT50, the addition of $34 \mathrm{wt} \%$ of MF led to a $54.6 \%$ reduction of PHRR and a 53.2\% decrease of THR, respectively. Moreover, TTPHRR increased to $85 \mathrm{~s}$ and FGR decreased to $0.07 \mathrm{~W} / \mathrm{s}$. This improvement is possible due to a gascondensed flame retardation mechanism that was created by interactions between MMT and MF in CNF aerogels. MMT acts as a catalyst, promoting the char formation of melamine. Meanwhile, diluting effect of $\mathrm{NH}_{3}$ gas from pyrolyzing $\mathrm{MF}$ decreases the concentration of fuel gas. Finally, a compact carbonaceous layer containing MMT formed on the top restricting the oxygen and heat propagation in materials. $^{[45]}$

\section{Conclusions}

We successfully fabricated highly flame retardant CNF-based aerogels with improved 
mechanical properties by mixing MF suspensions with CNF/MMT suspensions followed by a freeze-drying process. MF resins formed cross-linking between CNFs after curing at $120^{\circ} \mathrm{C}$. Cross-linking did not significantly affect the structures of the treated aerogels, but the process did improve their mechanical properties. When 50 $\mathrm{wt} \%$ of MF was added to the aerogels, the compression moduli and compressive stress $(\varepsilon=70 \%)$ were increased by 3.16 and 1.14 times, respectively. Introduction of MMT to MF cross-linked CNF aerogels raised the maximum decomposition temperature by nearly $20{ }^{\circ} \mathrm{C}$ and increased the materials' LOI values to levels as high as $57 \%$. This synergistic effect between MMT and MF on the flame retardant properties of CNFs aerogels was also proved via cone calorimetry. In CNF/MMT composite aerogels, $34 \mathrm{wt} \%$ of MF introduction led to a $54.6 \%$ reduction of PHRR and $53.2 \%$ decrement of THR, respectively due to a gas-condensed flame retardation mechanism. The prepared green CNF composite aerogels had excellent mechanical properties as well as high flame retardancy, opening the door to a wide range of potential applications.

\section{Acknowledgements}

This work was financially supported by the National Natural Science Foundation of China (Grant No.51573133) and Tianjin Education Ministry S\&T Project (2017KJ069) as well as Program for New Century Excellent Talents in University (NCET-12-1063). Miguel Sánchez-Soto acknowledges the financial support given by the Spanish Government through the project MAT2016-80045-R (AEI/FEDER,UE). 


\section{Conflict of Interest}

The authors declare no conflict of interest.

\section{Keywards}

Aerogels, cellulose nanofibrils, flame retardant, melamine-formaldehyde, montmorillonite.

\section{References}

[1] S. S. Kistler, Nature. 1931, 127, 741.

[2] N. Husing, U. Schubert, Angew. Chem. Int. Ed. 1998, 37, 22.

[3] D. W. Schaefer, K. D. Keefer, Phys. Rev. Lett. 1986, 56, 2199.

[4] C. Moreno-Castilla, F. J. Maldonado-Hódar, Carbon. 2005, 43, 455.

[5] J. Mao, J. Iocozzia, J. Huang, K. Meng, Y. Lai, Z. Lin, Energy Environ. Sci. 2018, 11,772 .

[6] J. Mao, M. Ge, J. Huang, Y. Lai, C. Lin, K. Zhang, K. Meng, Y. Tang, J. Mater. Chem. A 2017, 5, 11873.

[7] A. Gash, J. Non-Cryst. Solids. 2001, 285, 22.

[8] V. S. Pradeep, D. G. Ayana, M. Graczyk-Zajac, G. D. Soraru, R. Riedel, Electrochim. Acta. 2015, 157, 41.

[9] S. Kuiper, A. Embrechts, H. A. Every, T. de Vries, L. C. de Smet, Macromol. Mater. Eng. 2013, 298, 868.

[10] X. Z. Xu, F. Liu, L. Jiang, J. Y. Zhu, D. Haageson, D. P. Wiesenborn, ACS Appl. Mater. Interfaces. 2013, 5, 2999.

[11] M. Jonoobi, J. Harun, A. P. Mathew, K. Oksman, Compos. Sci. Technol. 2010, 70, 1742 .

[12] S. Sulaiman, N. L. Cieh, M. N. Mokhtar, M. N. Naim, S. M. M. Kamal, Process. Biochem. 2017, 55, 85.

[13] C. Aulin, J. Netrval, L. Wågberg, T. Lindström, Soft Matter. 2010, 6, 3298. 
[14] J. T. Korhonen, M. Kettunen, R. H. A. Ras, O. Ikkala, ACS Appl. Mater. Interfaces. 2011, 3, 1813.

[15] J. Zhao, C. Lu, X. He, X. Zhang, W. Zhang, ACS Appl. Mater. Interfaces. 2015, $7,2607$.

[16] R. Xiong, C. H. Lu, Y. R. Wang, Z. H. Zhou, X. X. Zhang, J. Mater. Chem. A. 2013, 1,14910 .

[17] Q. F. Zheng, H. L. Zhang, H. Y. Mi, Z. Y. Cai, Z. Q. Ma, S. Q. Gong, Nano Energy. 2016, 26, 504.

[18] H. B. Zhao, M. Chen, H. B. Chen, ACS Sustainable Chem. Eng. 2017, 5, 7012.

[19] B. Yuan, J. M. Zhang, Q. Y. Mi, J. Yu, R. Song, J. Zhang, ACS Sustainable Chem. Eng. 2017, 5, 11117.

[20] A. Javadi, Q. Zheng, F. Payen, A. Javadi, Y. Altin, Z. Cai, R. Sabo, S. Gong, ACS Appl. Mater. Interfaces. 2013, 5, 5969.

[21] L. Yang, A. Mukhopadhyay, Y. Jiao, Q. Yong, L. Chen, Y. Xing, J. Hamel, H. Zhu, Nanoscale 2017, 9, 11452.

[22] Y. Y. Han, X. X. Zhang, X. D. Wu, C. H. Lu, ACS Sustainable Chem. Eng. 2015, 3, 1853.

[23] B. Yuan, J. M. Zhang, J. Yu, R. Song, Q. Y. Mi, J. S. He, J. Zhang, Sci. China Chem. 2016, 59, 1.

[24] F. Laoutid, L. Bonnaud, M. Alexandre, J. M. Lopez-Cuesta, P. Dubois, Mat. Sci. Eng. R. 2009, 63, 100.

[25] H. W. Yan, J. L. Wei, B. Yin, M. B. Yang, Polym. Bull. 2015, 72, 2725.

[26] K. Wu, L. Song, Z. Z. Wang, Y. Hu, Polym. Advan. Technol. 2010, 19, 1914.

[27] R. C. Wilson, W. F. Pfohl, Vib. Spectrosc. 2000, 23, 13.

[28] L. Wang, M. Sánchez-Soto, T. Abt, Ind. Crop. Prod. 2016, 91, 15.

[29] X. F. Zhang, Y. Wang, J. Q. Zhao, M. J. Xiao, W. Zhang, C. H. Lu, ACS Sustainable Chem. Eng. 2016, 4, 4321.

[30] G. Socrates, Infrared and Raman Characteristic Group Frequencies: Tables and Charts. Third ed. Wiley Chichester. New York, 2001.

[31] P. S. Nayak, B. K. Singh, Bull. Mater. Sci. 2007, 30, 235. 
[32] C. Devallencourt, J. M. Saiter, A. Fafet, E. Ubrich, Thermochim. Acta. 1995, 259, 143.

[33] Y. S. Wu, Y. Li, L. Qin, F. L. Yang, D. C. Wu, J. Mater. Chem. B. 2013, 1, 204.

[34] Y. Huang, F. N. Jones, Prog. Org. Coat. 1996, 28, 133.

[35] Y. Jiang, W. Zhou, M. Jiang, P. Liu, J. Xu, Polym. Degrad. Stabil. 2017, 144, 331.

[36] C.Q. Yang, W. Wu, Y. Xu. Fire Mater. 2005, 29, 109.

[37] Y. T. Wang, S. F. Liao, K. Shang, M. J. Chen, J. Q. Huang, Y. Z. Wang, D. A. Schiraldi, ACS Appl. Mater. Interfaces. 2015, 7, 1780.

[38] S. Deville, E. Saiz, R. K. Nalla, A. P. Tomsia, Science. 2006, 311, 515.

[39] K. Shang, J. C. Yang, Z. J. Cao, W. Liao, Y. Z. Wang, D. A. Schiraldi, ACS Appl. Mater. Interfaces. 2017, 9, 22985.

[40] H. B. Chen, B. S. Chiou, Y. Z. Wang, D. A. Schiraldi, ACS Appl. Mater. Interfaces. 2013, 5, 1715.

[41] K. Shang, D. D. Ye, A. H. Kang, Y. T. Wang, W. Liao, S. M. Xu, Y. Z. Wang, Polymer. 2017, 131, 111.

[42] C. L. He, J. Y. Huang, S. H. Li, K. Meng, L. Y. Zhang, Z. Chen, Y. K. Lai, ACS Sustainable Chem. Eng. 2018, 6, 927.

[43] L. Wang, M. Sánchez-Soto, M. L. Maspoch, Mater. Design. 2013, 52, 609.

[44] T. Kashiwagi, R. H. Harris Jr, X. Zhang, R. Briber, B. H. Cipriano, S.R. Raghavan, W. H. Awad, J. R. Shields, Polymer. 2004, 45, 881.

[45] Y. Liua, J. S. Wanga, C. L. Deng, Polym. Advan. Technol. 2010, 21, 789. 


\section{Figure Captions:}

Figure 1. Scheme illustrating the procedure used to synthesize CNF/MMT/MF composite aerogels.

Figure 2. (a) FTIR spectra of pure CNF aerogels and composite aerogels; (b) Proposed scheme of cross-linking reaction of CNF and MF.

Figure 3. SEM micrographs of pure CNF aerogels and CNF composite aerogels (a)

CNF100; (b) CNF66MF34; (c) CNF50MF50; (d) CNF40MF60; (e) CNF50MMT50;

(f) CNF40MMT40MF20; (g) CNF33MMT33MF34; (h) CNF28MMT28MF44.

Figure 4. Compressive stress-strain curves of (a) pure CNF aerogels and MF crosslinked CNF aerogels and (b) Cross-linked CNF/MMT composite aerogels Figure 5. TGA (a) and DTG (b) curves of pure CNFs based aerogels.

Figure 6. (a) LOI values of pure CNF aerogels and modified CNF composite aerogels; (b) Images of CNF100 and CNF33MMT33MF34 samples after different time of ignition by an alcohol burner.

Figure 7. Heat release rate (a) and total heat release (b) of pure CNF aerogels and modified CNF aerogels. 
Table 1. Composition of the CNF/MMT/MF composite aerogel produced for this study.

\begin{tabular}{lcccc}
\hline Samples & $\begin{array}{c}\text { CNFs } \\
{[\mathbf{g}]}\end{array}$ & $\begin{array}{c}\text { MMT } \\
\text { [g] }\end{array}$ & $\begin{array}{c}\text { MF } \\
{[\mathbf{g}]}\end{array}$ & $\begin{array}{c}\text { DI water } \\
{[\mathbf{m L}]}\end{array}$ \\
\hline CNF100 & 1 & 0 & 0 & 100 \\
CNF66MF34 & 1 & 0 & 0.5 & 100 \\
CNF50MF50 & 1 & 0 & 1 & 100 \\
CNF40MF60 & 1 & 0 & 1.5 & 100 \\
CNF50MMT50 & 1 & 1 & 0 & 150 \\
CNF40MMT40MF20 & 1 & 1 & 0.5 & 150 \\
CNF33MMT33MF34 & 1 & 1 & 1 & 150 \\
CNF28MMT28MF44 & 1 & 1 & 1.5 & 150 \\
\hline
\end{tabular}


Table 2. Apparent densities and mechanical properties of CNF based aerogels.

\begin{tabular}{lcccccc}
\hline Samples & $\begin{array}{c}\rho \\
{\left[\mathbf{g} / \mathbf{c m}^{3}\right]}\end{array}$ & $\begin{array}{c}\boldsymbol{\sigma}_{\mathbf{1 0} \%} \\
{[\mathbf{k P a}]}\end{array}$ & $\begin{array}{c}\boldsymbol{\sigma}_{\mathbf{7 0}} \\
{[\mathbf{k P a}]}\end{array}$ & $\begin{array}{c}\boldsymbol{\sigma}_{\mathbf{s y}} \\
{\left[\mathbf{k P a} / \mathbf{g ~ c m} \mathbf{~ c m}^{-3}\right]}\end{array}$ & $\begin{array}{c}\mathbf{E} \\
{[\mathbf{k P a}]}\end{array}$ & $\begin{array}{c}\text { Es } \\
{\left[\mathbf{k P a} / \mathbf{g} \mathbf{~ c m}^{-3}\right]}\end{array}$ \\
\hline CNF100 & 0.0176 & $5.30 \pm 1.02$ & $65.15 \pm 9.71$ & $3701.70 \pm 247.72$ & $65.59 \pm 8.99$ & $3726.70 \pm 216.62$ \\
CNF66MF34 & 0.0206 & $8.68 \pm 1.81$ & $126.59 \pm 11.36$ & $6145.15 \pm 426.96$ & $103.59 \pm 9.23$ & $5028.64 \pm 448.98$ \\
CNF50MF50 & 0.0225 & $17.29 \pm 2.44$ & $159.32 \pm 14.65$ & $7080.89 \pm 138.92$ & $273.15 \pm 28.43$ & $12140.00 \pm 500.32$ \\
CNF40MF60 & 0.0208 & $12.96 \pm 1.84$ & $118.32 \pm 14.84$ & $5688.46 \pm 311.40$ & $177.13 \pm 12.83$ & $8515.87 \pm 164.20$ \\
CNF50MMT50 & 0.0168 & $12.45 \pm 1.35$ & $77.60 \pm 3.28$ & $4619.05 \pm 195.26$ & $165.89 \pm 34.97$ & $9874.40 \pm 442.42$ \\
CNF40MMT40MF20 & 0.0168 & $21.73 \pm 1.64$ & $80.84 \pm 4.04$ & $4811.90 \pm 240.49$ & $247.93 \pm 12.25$ & $14757.74 \pm 729.42$ \\
CNF33MMT33MF34 & 0.0170 & $21.70 \pm 1.55$ & $93.50 \pm 9.06$ & $5500.00 \pm 267.36$ & $309.06 \pm 22.59$ & $18180.00 \pm 623.59$ \\
CNF28MMT28MF44 & 0.0189 & $16.23 \pm 1.20$ & $50.68 \pm 2.82$ & $2681.48 \pm 164.27$ & $85.58 \pm 18.70$ & $4528.04 \pm 312.40$ \\
\hline
\end{tabular}


Table 3. TGA data of CNF based aerogels.

\begin{tabular}{lcccc}
\hline Samples & $\begin{array}{c}\mathbf{T}_{\mathbf{d} 5 \%} \\
{\left[{ }^{\mathbf{0}} \mathbf{C}\right]}\end{array}$ & $\begin{array}{c}\mathbf{T}_{\mathbf{d m a x}} \\
{\left[{ }^{\mathbf{0}} \mathbf{C}\right]}\end{array}$ & $\begin{array}{c}\mathbf{d W} / \mathbf{d T} \\
{\left[\mathbf{\%} /{ }^{\mathbf{0}} \mathbf{C}\right]}\end{array}$ & $\begin{array}{c}\mathbf{W}_{\mathbf{R}} \\
{[\mathbf{\%}]}\end{array}$ \\
\hline CNF100 & 253.17 & 288.5 & 9.2 & 35.5 \\
CNF66MF44 & 230.24 & 308.3 & 5.1 & 43.2 \\
CNF50MF50 & 222.15 & 309.7 & 4.0 & 40.9 \\
CNF40MF60 & 227.24 & 308.9 & 4.4 & 42.9 \\
CNF50MMT50 & 262.10 & 283.5 & 3.9 & 62.5 \\
CNF40MMT40MF20 & 262.55 & 307.8 & 3.4 & 60.6 \\
CNF33MMT33MF34 & 263.18 & 308.4 & 3.1 & 60.7 \\
CNF28MMT28MF44 & 249.26 & 307.4 & 2.9 & 57.7 \\
\hline
\end{tabular}


Table 4. UL-94 test results of pure CNF aerogels and modified CNF aerogels.

\begin{tabular}{lc}
\hline Samples & UL-94 \\
\hline CNF100 & NR \\
CNF66MF44 & NR \\
CNF50MF50 & NR \\
CNF40MF60 & NR \\
CNF50MMT50 & NR \\
CNF40MMT40MF20 & V-0 \\
CNF33MMT33MF34 & V-0 \\
CNF28MMT28MF44 & V-0 \\
\hline
\end{tabular}


Table 5. Combustive parameters of pure CNF aerogels and modified CNF aerogels.

\begin{tabular}{lccccc}
\hline Samples & $\begin{array}{c}\text { PHRR } \\
{[\mathbf{k W} / \mathbf{m} 2]}\end{array}$ & $\begin{array}{c}\text { TTPHRR } \\
{[\mathbf{s}]}\end{array}$ & $\begin{array}{c}\text { THR } \\
{[\mathbf{M J} / \mathbf{m} 2]}\end{array}$ & $\begin{array}{c}\text { FGR } \\
{[\mathbf{W} / \mathbf{s}]}\end{array}$ & $\begin{array}{c}\mathbf{W}_{\mathbf{R}} \\
{[\mathbf{\%}]}\end{array}$ \\
\hline CNF100 & 51.05 & 10.00 & 1.75 & 5.11 & 45.45 \\
CNF66MF34 & 22.51 & 10.00 & 2.48 & 2.25 & 32.00 \\
CNF50MF50 & 35.04 & 10.00 & 2.50 & 3.35 & 35.71 \\
CNF50MMT50 & 13.20 & 15.00 & 1.54 & 0.88 & 45.45 \\
CNF40MMT40MF20 & 13.17 & 10.00 & 0.74 & 1.32 & 62.50 \\
CNF33MMT33MF34 & 5.99 & 85.00 & 0.72 & 0.07 & 63.89 \\
\hline
\end{tabular}




\section{Highly Flame Retardant Melamine-formaldehyde Cross-linked \\ Cellulose Nanofibrils/Sodium Montmorillonite Aerogels with \\ Improved Mechanical Properties}

Table of Content:

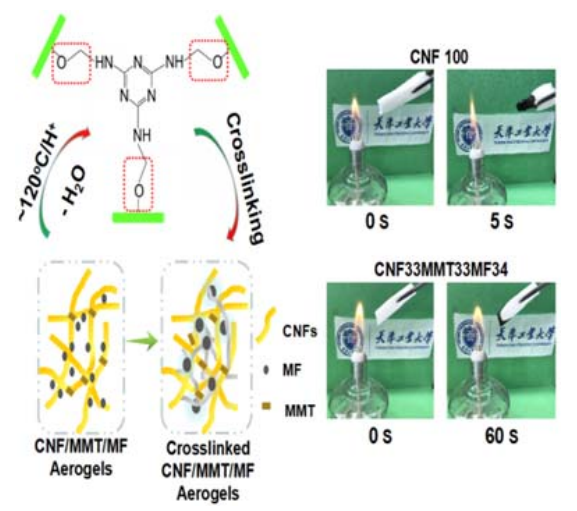

A facile method is proposed to fabricate nonflammable cellulose nanofibril/sodium montmorillonite composite aerogels with improved mechanical properties by crosslinking with melamine-formaldehyde. Montmorillonite and melamine-formaldehyde play a synergistic effect on the improvement of flame retardancy and mechanical properties of the CNFs aerogels. The modified cellulose nanofibril based aerogels have a limiting oxygen value as high as $57 \%$. 